\title{
Genetic Algorithm to Detect Different Sizes' Communities from Protein-Protein Interaction Networks
}

\author{
Marwa Ben M'Barek ${ }^{1,3}$ [a , Amel Borgi ${ }^{1,2}$, Sana Ben Hmida ${ }^{3}{ }^{\mathrm{b}}$ and Marta Rukoz ${ }^{3}$ \\ ${ }^{1}$ LIPAH, Faculté des Sciences de Tunis, Université de Tunis El Manar2092, Tunis, Tunisia \\ ${ }^{2}$ Institut Supérieur d'Informatique, Université de Tunis El Manar, 1002, Tunis, Tunisia \\ ${ }^{3}$ LAMSADE CNRS UMR 7243, Paris Dauphine University, PSL Research University, \\ Place du Maréchal de Lattre de Tassigny, Paris, France
}

\begin{abstract}
Keywords: Community Detection, Genetic Algorithm, Semantic Similarity, Protein-Protein or Gene-Gene Interaction Networks, Gene Ontology.

Abstract: The community detection in large networks is an important problem in many scientific fields ranging from Biology to Sociology and Computer Science. In this paper, we are interested in the detection of communities in the Protein-protein or Gene-gene Interaction (PPI) networks. These networks represent protein-protein or gene-gene interactions which corresponds to a set of proteins or genes that collaborate at the same cellular function. The goal is to identify such communities from gene annotation sources such as Gene Ontology. We propose a Genetic Algorithm based approach to detect communities having different sizes from PPI networks. For this purpose, we use a fitness function based on a similarity measure and the interaction value between proteins or genes. Moreover, a specific solution for representing a community and a specific mutation operator are introduced. In the computational tests carried out in this work, the introduced algorithm achieved excellent results to detect existing or even new communities from Protein-protein or Gene-gene Interaction networks.
\end{abstract}

\section{$1-$ INTRODUCTION}

Community detection in networks is one of the most popular topics of modern network science (Fortunato and Hric, 2016). It deals with an interesting computational technique for the analysis of networks. It can yield useful insights into the structural organization of a network and can serve as a basis for understanding the correspondence between structure and function (specific to the domain of the network). Identifying the community structure allows us to obtain some important information about the relationship and interaction among nodes.

In this paper, we are interested in detecting communities in biological networks. These networks have received much attention in the past few years since they model the complex interactions occurring among different components in the cell (Pizzuti and Rombo, 2014). We mainly focus on Protein-protein or Gene-gene interaction networks known as PPI networks. Their nodes correspond to proteins or genes and the edges correspond to pairwise interactions between genes or proteins. These communities give us an idea about the perception of the network's structure. The ultimate goal in biology is to determine how genes or proteins encode function in the cell. This work is multidisciplinary as it brings the field of biology and computer science in the broad sense.

Thus, the goal is to find communities having a biological sense (that participate in the same biological processes or that perform together specific biological functions) from gene annotation sources. To make this task, we have combined three levels of information:

1. Semantic level: information contained in biological ontologies such as Gene Ontology GO (Ashburner et al., 2000) and information obtained by the use of a similarity measure such as GS2 (Ruths et al., 2009), it assess the semantic similarity between proteins or genes.

(D) https://orcid.org/0000-0002-8307-3533

b(D) https://orcid.org/0000-0003-4202-613X 
2. Functional level: information contained in public databases describing the interactions of proteins or genes such as Search Tool for Recurring Instances of Neighbouring Gene (STRING) database (Mering et al., 2003).

3. Networks level: information contained in pathway databases that present community of proteins or genes such as KEGG database (Kanehisa and Goto, 2000).

In (Guo et al., 2006), the performed tests revealed that genes or proteins in the same community of the biological pathway database KEGG are semantically similar and are interacting. From this affirmation, we have proposed to take into account the similarity between proteins or genes that are annotated by terms of Gene Ontology (GO). In a previous work, we have tested different similarity measures to determine the most suitable one for this problem that is GS2 (Ben M'barek et al., 2018).

A lot of research effort has been put into community detection in different academic fields such as physics, mathematics and computer science. Meanwhile, various algorithms based on Genetic Algorithms (GA) have been proposed. These algorithms are used to overcome some drawbacks such as scaling up of network size. Indeed some of the community detection algorithms are unsuitable for very large networks and require a priori knowledge about the community structure, as the number and the size of communities which is not easy or impossible to obtain in real-world networks (Tasgin et al., 2007). The algorithms based on GA are very effective for community detection especially in very large complex networks (Jia et al., 2012). However, the vast majority of optimization methods proposed to detect community in PPI networks only use graph topology and do not use similarity measures between proteins or genes (Pizzuti and Rombo, 2014).

This paper presents a new community detection algorithm in PPI networks based on Genetic Algorithm (GA). This work is a generalization of a previous method that extract communities of a fixed size (Ben M'barek et al., 2018). Therefore, we propose a GA based approach that allows to find communities having different sizes. Alike the previous algorithm, the proposed method uses the similarity measures between proteins or genes and tries to find the best community structure by maximizing the concept of community measure. It is different from the community score introduced by Pizzuti which is not related to the density of groups (Pizzuti, 2008). Our measure is based on semantic similarity and interaction between proteins or genes. Moreover, we propose a new genetic operation that is a specific mutation operator adapted to our problem. The algorithm outputs the final community structure by selectively exploring the search space. Experiments on real datasets show the ability of the proposed approach to correctly detect communities having different sizes.

The contents are organized in six main sections. The next section provides the necessary background of literature survey to community's detection methods. Section 3 describes the biological field and the data used to formalize the problem. Section 4 depicts our main proposed algorithm for community detection. In section 5, experimental results on real data sets are presented and analyzed. Finally, section 6 draws the conclusion.

\section{COMMUNITY DETECTION METHODS}

This section presents a brief overview of the existing community detection algorithms. In recent years, many authors have given contributions to detect communities in large networks. The literature survey is divided into two categories: community detection on the basis of analytical approaches and those based on evolutionary approaches (Pizzuti, 2018) .

Analytical community methods firstly split networks into subgroups according to their topological characteristics, then the modularity assessment is carried out. The most famous and prominent algorithms in the literature have been presented by Girvan and Newman (Girvan and Newman, 2002; Newman and Girvan, 2004). Girvan and Newman have proposed the modularity assessment criterion, which is one of the most important assessment criteria in the community detection problem. According to the Girvan and Newman algorithm, the betweenness score (the number of shortest paths between all vertex pairs that run along the edge) is computed for all edges in the network in the first stage. In the second stage, the edge that has the maximum betweenness score is removed, and betweenness score is computed again for the all affected edges. In the final stage, the previous two stages are repeated until there are no more edges in the network.

Analytical algorithms do not reach the expected successful results in community detection from complex networks. Therefore, various optimization based algorithms have been proposed to provide different approaches to solve the community detection problem (Atay et al., 2017). With the 
modularity criterion, the community detection problem has become an optimization problem. Since 2002, several methods that divide networks into clusters according to the modularity criterion have been developed (Atay et al., 2017) .

In (Tasgin and Bingol, 2006) and (Liu et al., 2007) the authors presented an approach based on a GA to optimize the network modularity introduced by Newman and Girvan (Girvan and Newman, 2002).

Recently, some studies have indicated that the optimization of modularity has a main disadvantage (Fortunato and Barthélemy, 2007). It can fail in finding communities smaller than a fixed scale, even if these communities are well defined. The scale depends on the total size of the network and the interconnection degree of the communities. This resolution limit can constitute a weakness for all those methods whose objective to optimize is modularity.

Pizzuti (Pizzuti, 2008) has proposed an algorithm named GA-Net and she has used a special assessment function called community score in addition to the existing modularity function. This score uses only graph topology.

Compared to analytical methods, evolutionary methods present a number of advantages (Pizzuti, 2018) :

- The number of communities is automatically determined during the search process.

- Domain-specific knowledge can be incorporated inside the method, such as biased initialization, or specific variation operators instead of random, allowing a more effective exploration of the state space of possible solutions.

- Being population-based models, they are naturally parallel and efficient implementations can be realized to deal with large size networks.

A lot of detecting communities methods based on evolutionary approaches use only graph topology and do not use semantic similarity between nodes (Pizzuti and Rombo, 2014). The new community detection algorithm proposed in this paper is based on GA. It tries to find the best community structure by maximizing the concept of community measure. This measure is based on the graph topology (interaction) and the semantic similarity between nodes.

\section{USED DATA}

To help understand this work, we define some terms that are important for our discussion.
- A biological pathway is a series of actions among molecules in a cell that leads to a certain product or a change in the cell (National Human Genome Research Institute (NHGRI), 2015). There are many types of biological pathways such as metabolic pathways or Gene-regulation pathways.

- A biological network is a multiple biological pathways interacting with each other, example of biological networks: PPI networks (National Human Genome Research Institute (NHGRI), 2015).

To get more information on PPI network's structure, we have combined three information's levels: semantic level, functional level and network level. In this section, we describe the used data acquiesced from different sources.

\subsection{Semantic Level: Gene Information}

\subsubsection{GO Vocabulary Structure}

GO provides an ontology of defined terms which represent gene product properties. It comprises over 19000 terms organized in three sub-ontologies:

- Cellular Component (CC): the parts of a cell or its extracellular environment;

- Molecular Function (MF): the elemental activities of a gene product at the molecular level, such as binding or catalysis;

- Biological Process (BP): operations or sets of molecular events with a defined beginning and end, pertinent to the functioning of integrated living units: cells, tissues, organs, and organisms.

In this work, we focus on the BP domain. For example, the gene product cytochrome $c$ can be described by the biological process terms oxidative phosphorylation and induction of cell death (Ashburner et al., 2000). The GO ontology is structured as a directed acyclic graph, and each term designs relationships to one or more other terms in the same domain, and sometimes to other domains. Each GO term within the ontology has a term name (which may be a word or string of words), a unique alphanumeric identifier (which start by $\boldsymbol{G O}:$ ), a definition with cited sources, and a namespace indicating the domain to which it belongs. Terms may also have synonyms (which are classed as being exactly equivalent to the term name, broader, narrower, or related), references to equivalent 
concepts in other databases, and comments on term meaning or usage. We cite above an example of a GO term representation.

id: GO:0006119
name: oxidative phosphorylation
namespace: biological_process
def: "The phosphorylation of ADP to ATP that
accompanies the oxidation of a metabolite through
the operation of the respiratory chain. Oxidation of
compounds establishes a proton gradient across the
membrane, providing the energy for ATP
synthesis."
subset: goslim_pir
synonym: "respiratory-chain phosphorylation"
xref: Wikipedia:Oxidative_phosphorylation
is_a: GO:0006091 ! generation of precursor
metabolites and energy
is_a: GO:0016310! phosphorylation
relationship: has_part GO:0015986 ! ATP
synthesis coupled proton transport

We use GO to obtain data related to the BP aspects. We precisely focus on the relationship "is-a" and "part of" in order to identify the inheritance relationship between GO terms. From this source, we have extracted the unique identifier and the name of all the terms related to the $\mathrm{BP}$ aspects.

\subsubsection{Gene Ontology Annotation GOA}

A GO annotation is a statement about the function of a particular gene. Each GO annotation consists of an association between a gene and a GO term (Hill et al., 2008). From this project, we get a set of GO annotation for each gene of BP. For example, the MEIKIN gene is identified by ID: 728637 and annotated by the following sets: "GO: 0007060", "GO: 0010789", "GO: 0016321", "GO: 0045143", "GO: 0051754".

\subsubsection{Semantic Similarity}

In (Guo et al., 2006), the authors state that genes of the same community are semantically similar and interact with each other. From this indication, we supposed that genes belonging to the same community are similar and tried to find the best similarity measure between genes.

A gene can be annotated with numerous GO terms. To determine the similarity between two genes, we need to use an approach allowing to compare sets of terms that annotate these genes. Thus, we can quantify the similarity between these sets. In literature, there are three main approaches for measuring semantic similarity between the objects of an ontology (Resnik, 2011; Wang et al., 2007). The first are node-based approaches: the main data sources are the nodes and their properties. One concept commonly used in these approaches is information content, which measures how specific and informative a term is. The most prevalent nodebased approaches are Resnik's (Resnik, 2011, Lin's (Lin, 1998), Rel (Schlicker et al., 2006) and Jiang and Conrath's (Jiang and Conrath, 1997) methods. They were originally developed for the WorldNet, and then applied to GO (Pesquita et al., 2009). The second family of approaches is edge-based approaches: they are based mainly on counting the number of edges in the graph path between any two terms. The most common technique selects either the shortest path or the average of all paths when more than one path exists (Wu and Palmer, 1994). Among this family of approaches, there is the method of Rada (Rada et al., 1989) and the one of Wu and Palmer (Wu and Palmer, 1994). The third family of approaches are hybrid ones: Wang and al. (Wang et al., 2007) developed a hybrid measure in which each edge is given a weight according to the type of relationship. For a given term $c_{1}$ and its ancestor $c_{a}$, the authors define the semantic contribution of $c_{a}$ to $c_{1}$, as the product of all edge weights in the "best" path from $c_{a}$ to $c_{1}$, where the "best" path is the one that maximizes the product. Semantic similarity between two terms is then calculated by summing the semantic contributions of all common ancestors to each of the terms and dividing by the total semantic contribution of each term's ancestors to that term. Ruths and al. (Ruths et al., 2009) proposed GS2 (GO-based similarity of gene sets), a novel GO-based measure of genes set similarity. The measure quantifies the similarity of the GO annotations among a set of genes by averaging the contribution of each all gene's GO terms and their ancestor terms with respect to the GO vocabulary graph.

In order to choose the adequate measure of similarity, we have performed several tests GS2 (BEN M'BAREK et al., 2018). The best results were obtained with the GS2 measure. So, we decided to use the measure GS2 to characterize the similarity between genes in the rest of our work. 


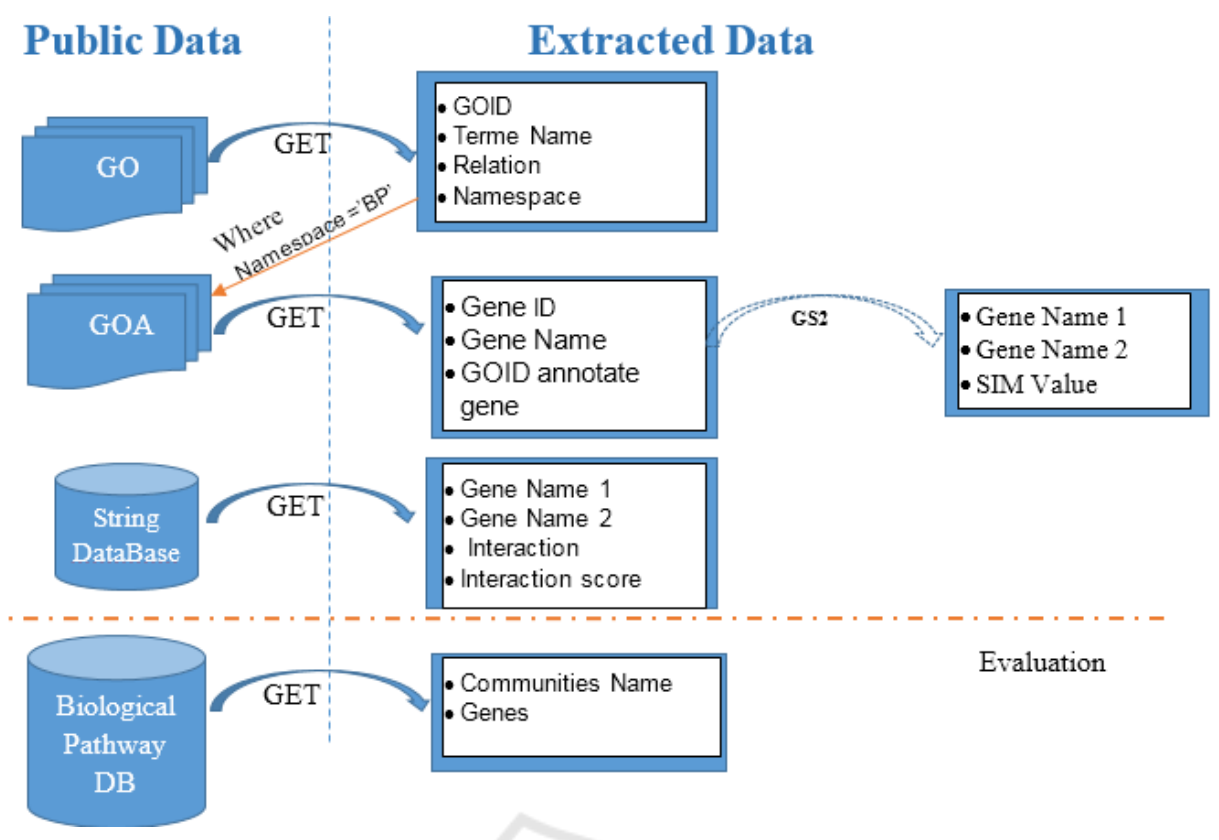

Figure 1: Summary of used data (Ben M'barek et al., 2018).

\subsection{Functional Level: Interaction between Genes}

To study the interaction between genes, we use the STRING (Search Tool for the Retrieval of Interacting Genes/Proteins) database. This database is a biological database and web resource of known and predicted protein-protein or gene-gene interactions. It contains information from several sources, including experimental data, computational prediction methods and public text collections (Mering et al., 2003; Snel et al., 2000). From this database, we extract the couples of genes or proteins that are interacting, the mode of interaction between these couple of genes and the interaction value which defines the number of citations of this interaction in the literature.

\subsection{Network Level: Biological Pathways Databases}

Among the various biological pathways databases, we cite those that we have used.

Reactome: is a free online database of human biological pathways and processes. The basic unit used to describe the data is the reaction (Croft et al., 2011).

Biocarta: catalogues community of several species. It makes it possible to visualize, construct or identify the networks mapping the known genomic and proteomic relationships. It offers a synthesis of these paths and represents them by graphs (Nishimura, 2001).

Ec number (Enzyme Commission Number): is a numerical classification scheme for enzymes (proteins that act as biological catalysts), based on the chemical reactions they catalyze. The chemical reaction catalyzed is the specific property that distinguishes one enzyme from another. It specify enzyme-catalysed reactions (Green and Karp, 2005) .

BBID (Biological Biochemical Image Database): is a WWW accessible relational database of archived image from different article that describe regulatory pathways. Pathway information is annoted and can be queried (Becker et al., 2000)

KEGG (Kyoto Encyclopedia of Genes and Genomes): is a knowledge base for systematic analysis of gene functions, linking genomic information with higher order functional information. The genomic information is stored in the GENES database, which is a collection of gene catalogs for all the completely sequenced genomes and some partial genomes with up-to-date annotation of gene functions (Kanehisa and Goto, 2000).

The biological pathway database used to test the proposed approach is KEGG as it was the one proposed by our biology expert. The other biological pathway databases are used to validate the experimental results as explained in section 5. In this database, we focused on the biological pathway which represent the communities name and the genes related to a community. 


\subsection{Summary of the Used Data}

Based on what has been illustrated from 3.1 to 3.3, the used data is summarized as:

- A gene or protein is described by an ID, a name and a set of terms that annotate it. For example, the MEKIN gene is presented as follows: ID: 728637 || NAME: MEIKIN || Annoted terms: [GO: 0007060, GO: 0010789, GO: 0016321, GO: 004514, GO: 0051754, GO: 0006119].

- Data related to the interaction between two genes. For example, the interaction between the HSPA1A gene and the GRPEL1 gene is: NameGene1: "HSPA1A" \| NameGene2: "GRPEL1» || Interaction: "reaction" \| Interaction Score: 900

- The biological pathway is described by a community name and a set of genes (pathway). These data are extracted from different pathway databases presented in subsection 3.3.

- The semantic similarity value computed by the GS2 method.

Figure 1 summarizes the sources of these extracted data. The first goal is to obtain information about a gene. Therefore, we get a set of GO terms that identify such gene from GO and GOA. Then, we acquire the interaction between couple of genes from STRING database.

We introduce the proposed approach to detect communities in the next section.

\section{GENETIC ALGORITHM FOR COMMUNITY DETECTION}

GAs have proved to be competitive alternative methods to traditional optimization and search techniques and they have been applied to many problems in diverse research and application areas such neural nets evolution, planning and scheduling, machine learning and pattern recognition(Goldberg, 1989; Petrowski and Ben-Hamida, 2017). Thus, it is also suitable for solving the community detection problem.

In this section, we describe the genetic algorithms used in this work as well as the genetic representation and operators.

The population consists of individuals that are the solutions of the problem. In our approach of protein or gene community detection, an individual is a set of proteins or genes that form a community having different sizes. To evaluate a solution, we propose a fitness function based on a community measure. The latter uses the similarity value and the interaction score of every pair of genes making up the solution. Moreover, we modify the steps of GA to satisfy the needs of our algorithm. Thus, we propose a new mutation operator, a clean-up phase and insert some additional steps during the population initialization. The algorithm works as follows:

1. Start with an initial population of communities having different sizes of a set of proteins or genes which may be generated randomly,

2. Select parents from the current population for mating,

3. Apply the crossover operator on the parents to generate new offsprings,

4. Clean up the new offsprings to eliminate the redundant genes after the crossover step,

5. Apply mutation operator on the new generated offsprings,

6. Evaluate these offsprings, and replace the worst existing individuals in the population by these offsprings,

7. Repeat the process from the second step while the stop condition is not satisfied (stop condition: number of generations is met or computation time reached).

The various steps of the GA are described in the following subsections.

\subsection{Genetic Representation}

One of the major important decisions to make while implementing a GA is deciding how to represent our individuals. A solution to our problem is a community of proteins or genes. We represent it by a vector T. In this representation each individual stores: the size of an individual that is the number of proteins or genes, the average value of similarity (see equation1), the average interaction score (see equation2) of each two genes or proteins and the list of the $n$ gens (each gene is represented by its name). This vector has $(n+3)$ elements where $\mathrm{n}$ is the size of a community, it corresponds to an individual in GA terms. Figure 2 illustrates the representation of an individual adopted in our algorithm.

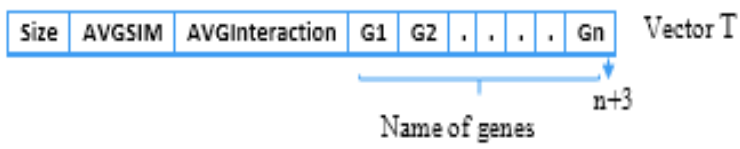

Figure 2: Example of individual representation designing a community. 
Table 1: Example of an initial population with five individuals.

\begin{tabular}{|c|c|c|c|c|c|c|c|c|c|c|}
\hline 5 & 0.553 & 0.398 & PDHA2 & MTHFD2L & RAC2 & GRHPR & ANAPC1 & & & \\
\hline 8 & 0.793 & 0.543 & ANAPC5 & SOS1 & CDC16 & AURKA & IL4 & ANAPC2 & ccNB2 & BUB1B \\
\hline 5 & 0.340 & 0.410 & RFK & HYI & GPI & UBC & IGF1R & & & \\
\hline 7 & 0.578 & 0.687 & HSD3878 & PFKP & LDHAL6B & FBXW7 & ACSM3 & MAX & & \\
\hline 6 & 0.632 & 0.591 & ALPP & BPGM & PLK1 & HK3 & HK1 & KEAP1 & & \\
\hline
\end{tabular}

$$
\operatorname{AVGSim}=\frac{\sum_{\mathrm{i}=1}^{\mathrm{n}-1} \operatorname{Sim}_{\mathrm{GS} 2}\left(\mathrm{G}_{\mathrm{i}}, \mathrm{G}_{\mathrm{i}+1}\right)}{\mathrm{n}}
$$

$$
\text { AVGInteraction }=\frac{\sum_{\mathrm{i}=1}^{\mathrm{n}-1} \text { InteractionValue }\left(\mathrm{G}_{\mathrm{i}}, \mathrm{G}_{\mathrm{i}+1}\right)}{\mathrm{n}}
$$

With:

- $\mathrm{n}$ : the size of a community.

- $\mathrm{Sim}_{\mathrm{GS} 2}$ : the similarity value between two genes, it is calculated using the semantic similarity measure GS2 (Ruths et al., 2009).

- InteractionValue : the score of an interaction between two genes extracted from STRING Database (Mering et al., 2003)

\subsection{Population Initialization}

The generation of the initial population in a GA is of profound importance since the characteristics of the initial solutions can affect the quality of the final solution as well as the running time of the algorithm. In this work, the population is defined as a twodimensional array of individuals. It is a set of individuals that represent some potential solutions of the problem. In order to initialize this population, we first randomly recover communities from the KEGG pathway database. Then, we randomly create the population with the recovered genes. The population is composed by individuals having different sizes. After that, we compute the similarity value using the GS2 measure and get the interaction score of each two genes of this group from the created "interaction" table. Next, we calculate the average similarity value and the average interaction score of each group forming this population. Table 1 presents an example of an initial population with five individuals having different sizes.

\subsection{Fitness Function}

The fitness function relates to the ability of the candidate to survive and reproduce. It takes as input a candidate solution to the problem and produces as output how better "fit" the solution is with respect to the considered problem. The computation of the fitness value is done repetitively in a GA and therefore it should be sufficiently fast. The choice of the fitness function is a critical step for obtaining good solutions. In the context of community detection the most popular function is modularity, originally introduced by Girvan and Newman (Girvan and Newman, 2002). In our work, we do not directly take into account the modularity, nevertheless the topological propriety of a community is taken into account through the interaction score between proteins or genes. Moreover, the fitness function is enriched with semantic information. Indeed, we used a defined fitness function based on the computation of the average similarity value and the average interaction score of each two genes existing in the community. We start from the hypothesis that genes in the same community are semantically similar and interact with each other. The fitness function is defined as follows (Ben M'barek et al., 2018):

$$
\mathrm{F}=\mathrm{W}_{1} \text { AVGSim }+\mathrm{W}_{2} \text { AVGInteraction }
$$

With:

- AVGSim and AVGInteraction defined in (1) and (2).

- $\mathrm{W}_{1}$ and $\mathrm{W}_{2}$ : weights $\in[0,1]$

We carried out tests with different values of $\mathrm{W}_{1}$ and $\mathrm{W}_{2}$. Then, we have taken the values which give the best results in terms of the number of known networks recovered from KEGG pathway database. The value taken for the fitness function are $\mathrm{W}_{1}=\mathrm{W}_{2}=$ 0.5 (Ben M'barek et al., 2018).

\subsection{Selection}

In this stage of a GA, individuals are selected from the population to be parents which mate and recombine to create offsprings for the next generation. Selection is very crucial to the convergence rate of the GA as good parents drives individuals to fitter solutions. The problem is how to select these individuals. In literature, there are many methods to select the best individuals such as roulette wheel selection, tournament selection, rank selection, elitism... (Goldberg and Deb, 1991). 


\begin{tabular}{l|l|l|l|l|l|l|l|l|} 
P1 & PDHA2 & MTHFD2L & RAC2 & GRHPR & ANAPC1 & & & \\
P2 & ANAPCS & SOS1 & CDC16 & AURKA & IL4 & ANAPC2 & CCNB2 & BUB1B \\
\hline Ch1 & PDHA2 & SOS1 & CDC16 & AURKA & ANAPC1 & & & \\
Ch2 & ANAPCS & MTHFD2L & RAC2 & GRHPR & IL4 & ANAPC2 & CCNB2 & BUB1B \\
\hline
\end{tabular}

Figure 3: Example of a two- point crossover operator.

In this work, we choosed the tournament selection method. In K-Way tournament selection, we select K individuals from the population at random and select the best out of these to become a parent. The same process is repeated for selecting the next parent. It is an extremely popular selection method in GA due to its efficiency and simple implementation (Goldberg and Deb, 1991).

\subsection{Genetic Operators}

After the generation of an initial population, a GA carries out the genetic operators to generate offspring based on the initial population. Once a new generation is created, the genetic process is performed iteratively until an optimal result is found or a maximum number of generations is met.

Crossover and mutation are two basic operators of GA. The performance of GA depends on them. These operators guide the algorithm towards a solution to a given problem. Their goal is to both exploit the best solutions and explore the search space.

For this work, we used the multi-point crossover: it is a generalization of the one-point crossover. Here two random sites are chosen with condition that the sites do not exceed the longest parent size. Then, the contents bracketed by these sites are exchanged between two mated parents to get two new offsprings. This operator is usually applied with a high probability (pc) (Pizzuti, 2018). To better understand this kind of crossover, a graphical illustration can be seen in Figure 3. In this example, two sites are chosen at random in position 1 and 4. Then two offspring $(\mathrm{ch} 1, \mathrm{ch} 2)$ are generated by exchanging the values of the selected parents (P1,P2).

The mutation is an operator that acts in a rarer fashion and in an unpredicted form to modify the genes of the individual, promoting the diversification of the population. However, the mutation must not be too destructive and nullify the process of finding an optimal solution (Pizzuti, 2018).

For mutation, we proposed a new operator that can better meet the objectives of our problem.
Mutation may be defined as a small random tweak in the individual, to get a new solution. It is used to maintain and introduce diversity in the population and is usually applied with a low probability (pm). If the probability is very high, the GA gets reduced to a random search (Pizzuti, 2018).

We present now a new mutation operator that is specific to our problem, it should allow a better exploration of the search space than the random mutation. Its goal is to maximize the chance of creating a better solution than the original one. This operator can integrate a new gene in order to replace a gene having a poor quality or to enlarge the size of the community. To mutate a solution $\mathrm{S}$, the mutation operator alters only one gene at a time and uses a score function, denoted GS, applied to each gene in $\mathrm{S}$. This score helps us to detect the gene having the best score in a community as well as the gene having the worst score. It is equal to the sum of the average similarity and the average interaction score of a gene in a community. It is defined as follows:

$$
\begin{aligned}
& \operatorname{AVGSim}(G)=\frac{\sum_{i=1}^{n} \operatorname{Sim}_{G S 2}\left(G, G_{i}\right)}{n-1} \\
& \operatorname{AVGInteraction}(G) \\
& =\frac{\sum_{i=1}^{n} \operatorname{IneractionValue}\left(G, G_{i}\right)}{n-1}
\end{aligned}
$$

$$
\mathrm{GS}(\mathrm{G})=\operatorname{AVGSim}(\mathrm{G})+\operatorname{AVGInteraction}(\mathrm{G})
$$

With:

- $\mathrm{G} \neq \mathrm{G}_{\mathrm{I}}$

- $\operatorname{Sim}_{\mathrm{GS} 2}\left(\mathrm{G}, \mathrm{G}_{\mathrm{i}}\right)$ : The similarity value of a gene $G$ compared to the other genes in the community.

- InteractionValue $\left(G, G_{i}\right)$ : The interaction score of a gene $G$ compared to the others in the community.

- $\mathrm{n}$ : size of an individual (community). 
The proposed mutation operator is applied according to the following steps:

1. Select in a solution $\mathrm{S}$ a gene having the highest score GS that will be called "bestGene".

2. Randomly search a gene $G^{\prime}$ from the "interaction" table with which the "bestGene" interacts and $\mathrm{G}^{\prime} \notin \mathrm{S}$.

3. Get the gene having the lowest score GS in S, it will be called "worstGene".

4. Fixe a threshold $\theta=0.5$

5. If the score of the "worstGene" $<=\theta$ then replace the "worstGene" by the gene selected in the second step.

6. Else insert into the end position of the solution the gene selected in the second step and update the size.

\section{EXPERIMENTAL RESULTS}

In this section, we study the effectiveness of our approach on real datasets (Pathways selected from KEGG Pathway database).

We first carried out tests to tune the GA parameters. Different parameters values were tested: generation number set at 100, 300 and 500, size of the population set at 10, 20,30, 70 and 100 , crossover rate set at $0.5,0.6, \ldots 1$ and mutation rate set at $0,01,0.1$, $0.2, \ldots, 0.5$. Based on these tests, we choose the combination of the parameters values giving the best results (highest values of fitness function), namely: population size 30 , generation number 100 , crossover rate 0.8 , and mutation rate 0.01 . Moreover, a weak value of mutation probability allows to prevent the algorithm to be blocked in a local minimum. Concerning the size of individual in the initial population, we fix it in the range of 5 to 40 .

In order to check the ability of our approach to successfully detect the community structure of a network, we use randomly selected proteins or genes that are present in communities from the reference pathway database KEGG. More precisely, our approach has been tested with five datasets proposed by our biological expert, in total we have 595 genes, as described in Table2. These datasets correspond to a real and existing communities and are collected from the KEGG pathway database.

The evaluation consists in verifying how the proposed method is likely to find gene or proteins communities existing in the KEGG pathway database. Actually, the tests showed that it was possible to detect communities of genes or proteins existing in KEGG database or a new community having high interaction and high similarity between its genes or proteins and that do not appear in KEGG.

Table 2: The used datasets.

\begin{tabular}{|c|c|}
\hline Datasets & $\begin{array}{c}\text { Number of } \\
\text { genes }\end{array}$ \\
\hline Apoptosis & 88 \\
\hline B cell receptor signalling pathway & 75 \\
\hline Purine metabolism & 159 \\
\hline Rna degradation & 159 \\
\hline Oocyte meiosis & 114 \\
\hline Total & 595 \\
\hline
\end{tabular}

We performed tests to determine communities having different sizes. We run our approach 20 times with proteins or genes chosen randomly from the five proposed datasets. And, we retained each time the best community. Thus, we have 20 best communities with diverse sizes varying from 5 to 40 . When a solution is found the question that arises is how it will be evaluated.

Our biology expert proposed to evaluate this community by checking if it exists in KEGG or other biological pathway databases. Each new community Rnew founded by our approach is presented to the DAVID tools (Database for Annotation Visualization and Integrated Discovery), which compares this community with others in different databases and gives the percentage of Rnew's genes that belong to the existing communities in those databases. DAVID bioinformatics resources consist of an integrated biological knowledge-base and analytic tools that aim at systematically extracting biological meaning from large gene/protein lists. It is the most popular functional annotation programs used by biologists (Sherman et al., 2007). It takes as input a list of genes and exploits the functional annotations available on these genes in a public database such as, KEGG Pathways in order to find common functions that are sufficiently specific to these genes. Table 3 below represents the minimum and the maximum percentage of genes that belong to the used communities of KEGG pathways. Comparing a community Rnew founded by our approach to the datasets used to create the initial population, allows to evaluate the ability of our method to rebuilt communities with the initial proteins or genes. 
Table 3: Communities' detection: experimental results compared to the proposed datasets.

\begin{tabular}{|c|c|c|}
\hline Datasets & $\begin{array}{c}\text { Percentage } \\
\text { Min }\end{array}$ & $\begin{array}{c}\text { Percentage } \\
\text { Max }\end{array}$ \\
\hline Apoptosis & $25 \%$ & $67 \%$ \\
\hline $\begin{array}{c}\text { B cell receptor } \\
\text { signaling pathway }\end{array}$ & $25 \%$ & $67 \%$ \\
\hline Purine metabolism & $50 \%$ & $100 \%$ \\
\hline RNA degradation & - & $29 \%$ \\
\hline Oocyte meiosis & $50 \%$ & $100 \%$ \\
\hline
\end{tabular}

From table 3, we find that obtained communities correspond to some "parts" of the real communities, and in some cases to a complete network (percentage $100 \%$ ). Therefore, the proposed algorithm able to efficiently rebuilt communities with the initial used proteins or genes. In a further work, additional tests should be conducted to confirm these results.

We evaluate also the obtained communities by checking if they exist in other biological pathway databases. The biological databases used to evaluate our results are Biocarta, Reactome, BBID and EC Number. We also used KEGG pathway database to compare the found communities by our approach with other communities than those used for the initialization step (datasets in table 2). The results of this evaluation are shown in table 4.

Table 4: Evaluation of new communities having different sizes.

\begin{tabular}{|c|c|c|}
\hline Pathway Databases & $\begin{array}{c}\text { Percentage } \\
\text { Min }\end{array}$ & $\begin{array}{c}\text { Percentage } \\
\text { Max }\end{array}$ \\
\hline BBid & $25 \%$ & $50 \%$ \\
\hline Biocarta & $20 \%$ & $66 \%$ \\
\hline Ec_number & $10 \%$ & $100 \%$ \\
\hline Reactome_pathway & $14 \%$ & $100 \%$ \\
\hline $\begin{array}{c}\text { Kegg_pathway (other } \\
\text { datasets ) }\end{array}$ & $15 \%$ & $100 \%$ \\
\hline
\end{tabular}

The results presented in table 4 show that the new communities obtained by our algorithm correspond to some "parts" of real networks existing in other biological pathway databases, and in some cases to a complete network (percentage 100\%). These results are considered very satisfactory by the biology expert. They constitute an initial validation of our algorithm and show the relevance of the used fitness function. These tests should be supplemented on a larger scale with other datasets and different communities.

Furthermore, we compare the results obtained by our new approach with the method proposed in (Ben M'barek et al., 2018) where the communities have a fixed predetermined size. We name this approach Ben M'Barek et al. 2018. The same datasets proposed by the biological expert (Table 2) and the same GA parameters were used for both approaches. The program was executed 20 times. Table 5 illustrates the results of our approach and the other method for identifying communities. Here again, we used the DAVID tools to estimate the recovery rate of the found communities with existing communities in different biological databases.

Table 5: Comparison of the proposed approach and the BEN M'barek et al.2018 approach for identifying communities.

\begin{tabular}{|c|c|c|c|c|}
\hline \multirow{2}{*}{ Pathway Databases } & \multicolumn{2}{|c|}{$\begin{array}{c}\text { Ben M'barek } \\
\text { et al.2018 } \\
\text { approach }\end{array}$} & \multicolumn{2}{c|}{$\begin{array}{c}\text { The proposed } \\
\text { apprach }\end{array}$} \\
\cline { 2 - 5 } & $\begin{array}{c}\% \\
\text { Min }\end{array}$ & $\begin{array}{c}\% \\
\text { Max }\end{array}$ & $\begin{array}{c}\% \\
\text { Min }\end{array}$ & $\begin{array}{c}\% \\
\text { Max }\end{array}$ \\
\hline BBid & $20 \%$ & $30 \%$ & $25 \%$ & $50 \%$ \\
\hline Biocarta & $20 \%$ & $60 \%$ & $20 \%$ & $66 \%$ \\
\hline Ec_number & $20 \%$ & $70 \%$ & $10 \%$ & $100 \%$ \\
\hline Reactome_pathway & $20 \%$ & $70 \%$ & $14 \%$ & $100 \%$ \\
\hline Kegg_pathway & $20 \%$ & $90 \%$ & $15 \%$ & $100 \%$ \\
\hline
\end{tabular}

Table 5 clearly shows the good performance of the new proposed approach to detect communities having different sizes with respect to Ben M'barek et al. 2018 approach to detect communities having the same sizes. Our new method achieves the highest percentage $100 \%$ in three pathway databases: Kegg, Reactome and Ec_number. For example, on the Kegg pathway database, the new proposed approach obtained the max percentage value $100 \%$ which corresponds to a complete network. The worst percentage value is of $15 \%$ which corresponds to some "parts" of the real communities.

To conclude, the obtained results show the capability of the proposed GA to effectively deal with community identification in networks. Further extensions will be proposed to detect networks with larger size and identify new networks not yet known int the public biological databases.

\section{CONCLUSIONS}

In this paper, we have proposed an approach based on GA to detect communities of interacting genes or proteins. This approach allows to find communities having different sizes, it is a generalization of the previous work here the found communities had a fixed predetermined size. Moreover, it introduces the concept of community measure and searches for optimal partitioning of the network by maximizing these measures. Our contribution in this paper is 
twofold. First, we applied GA to community detection in PPI networks. Second, we defined a specific mutation operator adapted to the considered biological problem. Dense communities existing in the network structure are obtained at the end of the algorithm by selectively exploring the search space, without the need to know in advance the community size. The experimental results showed the ability of our approach to correctly detect communities having different sizes. Future research will aim at modifying the proposed fitness function for example by adding the modularity value and applying multi-objective optimization to improve the quality of the results.

\section{ACKNOWLEDGEMENTS}

We would like to show our gratitude to Dr. Walid BEDHIAFI (Laboratoire de Génétique Immunologie et Pathologies Humaines, Université de Tunis El Manar ) for assistance to comprehend the biological fields and for the interpretation of the results.

\section{REFERENCES}

Ashburner, M., Ball, C.A., Blake, J.A., Botstein, D., Butler, H., Cherry, J.M., Davis, A.P., Dolinski, K., Dwight, S.S., Eppig, J.T., Harris, M.A., Hill, D.P., Issel-Tarver, L., Kasarskis, A., Lewis, S., Matese, J.C., Richardson, J.E., Ringwald, M., Rubin, G.M., Sherlock, G., 2000. Gene ontology: tool for the unification of biology. The Gene Ontology Consortium. Nat. Genet. 25, 25-29.

Atay, Y., Koc, I., Babaoglu, I., Kodaz, H., 2017. Community detection from biological and social networks: A comparative analysis of metaheuristic algorithms. Applied Soft Computing 50, 194-211.

Becker, K.G., White, S.L., Muller, J., Engel, J., 2000. BBID: the biological biochemical image database. Bioinformatics 16, 745-746.

Ben M'barek, M., Borgi, A., Bedhiafi, W., Ben Hmida, S., 2018. Genetic Algorithm for Community Detection in Biological Networks. Procedia Computer Science, Knowledge-Based and Intelligent Information \& Engineering Systems: Proceedings of the 22nd International Conference, KES-2018, Belgrade, Serbia 126, 195-204.

Croft, D., O’Kelly, G., Wu, G., Haw, R., Gillespie, M., Matthews, L., Caudy, M., Garapati, P., Gopinath, G., Jassal, B., Jupe, S., Kalatskaya, I., Mahajan, S., May, B., Ndegwa, N., Schmidt, E., Shamovsky, V., Yung, C., Birney, E., Hermjakob, H., D’Eustachio, P., Stein, L., 2011. Reactome: a database of reactions, pathways and biological processes. Nucleic Acids Res. 39, D691-697.

Fortunato, S., Hric, D., 2016. Community detection in networks: A user guide. Physics Reports 659, 1-44.
Girvan, M., Newman, M.E.J., 2002. Community structure in social and biological networks. Proc. Natl. Acad. Sci. U.S.A. 99, 7821-7826.

Goldberg, D. E., 1989. Genetic Algorithms in Search, Optimization and Machine Learning, 1st ed. AddisonWesley Longman Publishing Co., Inc., Boston, MA, USA.

Goldberg, D. E., Deb, K., 1991. A comparative analysis of selection schemes used in genetic algorithms, in: Foundations of Genetic Algorithms. Morgan Kaufmann, pp. 69-93.

Green, M. L., Karp, P.D., 2005. Genome annotation errors in pathway databases due to semantic ambiguity in partial EC numbers. Nucleic Acids Res 33, 4035-4039.

Guo, X., Liu, R., Shriver, C.D., Hu, H., Liebman, M.N., 2006. Assessing semantic similarity measures for the characterization of human regulatory pathways. Bioinformatics 22, 967-973.

Hill, D.P., Smith, B., McAndrews-Hill, M.S., Blake, J.A., 2008. Gene Ontology annotations: what they mean and where they come from. BMC Bioinformatics 9, S2.

Jia, G., Cai, Z., Musolesi, M., Wang, Y., Tennant, D.A., Weber, R.J.M., Heath, J.K., He, S., 2012. Community Detection in Social and Biological Networks Using Differential Evolution, in: Hamadi, Y., Schoenauer, M. (Eds.), Learning and Intelligent Optimization, Lecture Notes in Computer Science. Springer Berlin Heidelberg, pp. 71-85.

Jiang, J. J., Conrath, D.W., 1997. Semantic Similarity Based on Corpus Statistics and Lexical Taxonomy. arXiv:cmp-lg/9709008

Kanehisa, M., Goto, S., 2000. KEGG: kyoto encyclopedia of genes and genomes. Nucleic Acids Res. 28, 27-30.

Lin, D., 1998. An Information-Theoretic Definition of Similarity. In Proceedings of the 15th International Conference on Machine Learning. Morgan Kaufmann, pp. 296-304.

Liu, X., Li, D., Wang, S., Tao, Z., 2007. Effective Algorithm for Detecting Community Structure in Complex Networks Based on GA and Clustering, in: Shi, Y., Albada, G.D. van, Dongarra, J., Sloot, P.M.A. (Eds.), Computational Science - ICCS 2007, Lecture Notes in Computer Science. Springer Berlin Heidelberg, pp. 657-664.

Mering, C. von, Huynen, M., Jaeggi, D., Schmidt, S., Bork, P., Snel, B., 2003. STRING: a database of predicted functional associations between proteins. Nucl. Acids Res. 31, 258-261.

National Human Genome Research Institute (NHGRI), 2015. Biological Pathways Fact Sheet. National Human Genome Research Institute (NHGRI).

Newman, M.E.J., Girvan, M., 2004. Finding and evaluating community structure in networks. Physical Review E 69.

Nishimura, D., 2001. BioCarta. Biotech Software \& Internet Report 2, 117-120.

Pesquita, C., Faria, D., Falcão, A.O., Lord, P., Couto, F.M., 2009. Semantic Similarity in Biomedical Ontologies. PLoS Comput Biol 5. 
Pizzuti, C., 2018. Evolutionary Computation for Community Detection in Networks: A Review. IEEE Transactions on Evolutionary Computation 22, 464483.

Pizzuti, C., 2008. GA-Net: A Genetic Algorithm for Community Detection in Social Networks. Presented at the International Conference on Parallel Problem Solving from Nature, Springer, Berlin, Heidelberg, pp. 1081-1090.

Petrowski, A., Ben-Hamida, S., 2017. Evolutionary Algorithms. John Wiley \& Sons.

Pizzuti, C., Rombo, S.E., 2014. Algorithms and tools for protein-protein interaction networks clustering, with a special focus on population-based stochastic methods. Bioinformatics 30, 1343-1352.

Rada, R., Mili, H., Bicknell, E., Blettner, M., 1989. Development and application of a metric on semantic nets. IEEE Transactions on Systems, Man, and Cybernetics 19, 17-30.

Resnik, P., 2011. Semantic Similarity in a Taxonomy: An Information-Based Measure and its Application to Problems of Ambiguity in Natural Language. arXiv:1105.5444 [cs].

Ruths, T., Ruths, D., Nakhleh, L., 2009. GS2: an efficiently computable measure of GO-based similarity of gene sets. Bioinformatics 25, 1178-1184.

Schlicker, A., Domingues, F.S., Rahnenführer, J., Lengauer, T., 2006. A new measure for functional similarity of gene products based on Gene Ontology. BMC Bioinformatics 7, 302.

Sherman, B. T., Huang, D. W., Tan, Q., Guo, Y., Bour, S., Liu, D., Stephens, R., Baseler, M.W., Lane, H.C., Lempicki, R. A., 2007. DAVID Knowledgebase: a gene-centered database integrating heterogeneous gene annotation resources to facilitate high-throughput gene functional analysis. BMC Bioinformatics 8, 426.

Snel, B., Lehmann, G., Bork, P., Huynen, M.A., 2000. STRING: a webserver to retrieve and display the repeatedly occurring neighbourhood of a gene. $\mathrm{Nucl}$. Acids Res. 28, 3442-3444.

Tasgin, M., Bingol, H., 2006. Community Detection in Complex Networks using Genetic Algorithm. arXiv:cond-mat/0604419.

Tasgin, M., Herdagdelen, A., Bingol, H., 2007. Community Detection in Complex Networks Using Genetic Algorithms. arXiv:0711.0491 [physics].

Wang, J.Z., Du, Z., Payattakool, R., Yu, P.S., Chen, C.-F., 2007. A new method to measure the semantic similarity of GO terms. Bioinformatics 23, 1274-1281.

Wu, Z., Palmer, M., 1994. Verbs Semantics and Lexical Selection. In: Proceedings of the 32Nd Annual Meeting on Association for Computational Linguistics, $A C L$ '94. Association for Computational Linguistics, Stroudsburg, PA, USA, pp. 133-138. 